\title{
LA HEIEROGÉNEA CONSTRUCCIÓN DEL SUFRIMIENTO EN EL GRAN BUENOS AIRES
}

\section{Pablo Semán \\ Universidad de Buenos Aires - Argentina}

Resumo: O presente trabalho descreve três concepções diferenciadas de doença, de cura e, consequentemente, da noção de pessoa. O trabalho foi feito uma área periférica da cidade de Buenos Aires. Os dados etnográficos centram na questão da trajetória terapêutica religiosa dos habitantes deste subúrbio. Agentes religiosos católicos e pentecostais, curandeiros, médicos e psicólogos fazem parte de um conjunto a partir do qual os sujeitos selecionam e combinam termos que dão consistência às seguintes concepções: a que chamaremos monista, baseada na estreita relação entre as categorias físicas e morais, a dualista que opera sobre a base da separação da ordem espiritual e material e a trinitária que integra uma esfera psicológica e implica uma reação contra a segunda e uma reabilitação parcial e original da primeira.

Abstract: This work describes three different ways of conceiving suffering and cure, and consequently, three different concepts of a personhood. The ethnographic work was done in a peripherical region of Buenos Aires metropolitan area. It focus in the inquire about the therapeutic and religious experiences of these people. Religious agents - pentecostal and catholics -, healers, doctors and psychologists are a set of common resources from wich this population selects and combines terms that are the basis of three different conceptions: the monist conception, based on the close relationship between the physical and the moral categories; the dualist conception, based on the spiritual and material distinction; and the trialist conception, wich includes a psychological element, a reaction against the dualist conception, and it aims a partial recuperation and an original reelaboration of the monist conception. 


\section{Introducción}

Dentro del orden que los estudios sobre la idea persona circunscriben se encuentran, sin duda, las divisiones bajo las cuales un hombre concibe a otro, las relaciones que se establecen entre esas partes y el estatuto de lo corporal en ese régimen. ${ }^{1}$ Tal como lo resalta Duarte (1986) el campo de sentidos que se imbrica alrededor de la idea de perturbación resulta revelador de la arquitectura del concepto de persona en juego en una cultura singular.

Teniendo en cuenta esta última premisa y las dimensiones arriba citadas este trabajo se propone dar cuenta de una constatación etnográfica: la coexistencia de tres concepciones diferenciadas sobre las divisiones constitutivas de la persona, en las prácticas y discursos sobre la enfermedad y la cura, entre miembros de diversos grupos religiosos de un suburbio del Gran Buenos Aires. Es justamente por la inserción religiosa de los informantes que será analizada, adicionalmente, otra dimensión: los diversos sentidos que adquiere la noción de milagro en los diferentes sistemas de representación la noción de milagro referida los procesos de cura. ${ }^{2}$

Duarte (1994) propone la recuperación de la expresión físico moral como referencia apropiada para contener el conjunto de las de la problematizaciones efectuadas por diversas tradiciones culturales en cuanto a los núcleos de la perturbación y el concepto de persona subyacente. Según esta indicación consideramos las tres nociones que presentaremos como formas diferenciales de articular simbólicamente la dimensión físico moral de la experiencia humana.

El criterio que divide a las diferentes concepciones atiende a las divisiones de la experiencia, las relaciones entre las partes que esos conceptos

\footnotetext{
Los presupuestos teóricos que nos guían remiten al valor analítico de la distinción entre el agente empírico y su valor culturalmente construido Dumont (1992).

2 Una hipótesis relativa a la confluencia de diferentes temporalidades culturales y, por lo tanto, diferentes conceptos de persona, en el conjunto del campo religioso puede encontrarse ha sido formulada por Sanchis (1997). La especificidad de nuestro planteo es que esta confluencia puede observarse ya en el segmento popular del campo religioso y en las consecuencias que esto tiene para la articulación de nociones que en principio pertenecen a la modernidad.
} 
establecen y las características que adquiere lo corporal en su contexto. ${ }^{3} \mathrm{La}$ primera de estas concepciones, a la que llamare monista, se basa en el uso de categorías de enfermedad y cura que reenvían a la estrecha unidad de las categorías físicas y morales. La segunda, a la que llamaremos dualista, se estructura sobre la base de la distinción radical entre lo material corporal y lo espiritual mientras que la última de estas concepciones, a la que llamaremos trinaria, sostiene una división que implica los planos de lo físico, lo psicológico y lo espiritual.

\section{I - Aproximación al contexto etnográfico}

Villa Libertad es un barrio localizado en la zona sur del Gran Buenos Aires en una zona de industrialización tardía en la que conviven habitantes radicados entre 1940 y 1960 con migrantes recientes de las provincias más pobres del interior de la Argentina y un importante porcentaje de extranjeros provenientes de Paraguay y Bolivia.

La mayor parte de los habitantes son trabajadores que formando parte de diversas categorías de trabajo (formal e informal, en dependencia y por cuenta propia) se encuentran entre las carnadas de renta más baja de la sociedad. La infraestructura de servicios del Barrio provee a sus habitantes de servicios básicos de salud y educación y es de destacar que la alfabetización es casi universal para las generaciones nacidas a partir de los años 30 .

El desarrollo de las instituciones religiosas en el barrio se caracteriza por la fragmentación de las alternativas en el campo religioso. De un lado pluralización de las alternativas religiosas encarnada en el crecimiento de las iglesias Pentecostales (y secundariamente de los Testigos de Jehová y los Mormones) desde inicios de los años 80. Del otro la fragmentación del campo católico entre diversos regímenes de práctica alimentados por distintos tipos de institucionalización: el catolicismo de los habitantes de los pueblos rurales reproducido en los hogares urbanos, los efectos racionalizantes de las políticas

\footnotetext{
Si bien esta categorización sigue posiciones expuesta por Duarte (1986), he preferido una nominación específica. Es que la base empíricas y los recursos analíticos aquí desplegados son mucho más elementales que los presentes en las citadas obras. Esto vale especialmente para la concepción monista que, sin dudas está contenida en una concepción holista y relacional de la persona.
} 
de evangelización posconciliares, la aparición y expansión del movimiento carismático. Finalmente debe considerarse la existencia de un denso campo de prácticas de cura que florece en una situación de frontera entre el catolicismo y la religiosidad popular autónoma. En él se combinan diversas tradiciones de cura, y lo unifican como espacio de lo que llamamos curanderos el uso, las representaciones de sus clientes, la red de usuarios en que se insertan y se vuelven intercambiables. Como correlato de esa situación la trayectoria de los sujetos en el campo religioso se caracteriza por la fluctuación intra e interdenominacional, y la reelaboración autónoma de la experiencia religiosa. En esa apropiación inciden no solo otras experiencias religiosas sino, también, las experiencias culturales desarrolladas en las redes familiares, las instituciones de salud y educación. Es en el contrapunto entre trayectorias personales y este fragmentado campo de instituciones religiosas y seculares que emergen, como producto de la elaboración individual y colectiva, las concepciones que a continuación reseñaremos.

\section{II - Monistas, dualistas, y trinarlos}

Monistas: Víctor tenía los siguientes problemas: le faltaban las "fuerzas para hacer las cosas”, no sabía si quedarse con su mujer o separarse y una serie sistemática de "mala suerte” le impedía obtener trabajo. Durante las semanas en que esta situación se le hacía evidente no tuvo dudas de que la serie de situaciones referidas formaban parte de un único mal (probable nota sobre que él disponía de otra clave para interpretar). Y para resolverlo acudió a lo de una curandera. Dialogando con ella y exponiéndose a sus poderes de diagnóstico ${ }^{4}$ supo lo que le pasaba: una mujer que quería que él se enamorara de ella encargo a otra curandera un trabajo que salió mal y que le "arruinó” la vida. ¿Cómo sucedió eso?. En la concepción que la curandera y Víctor se forjaron acerca de los hechos esto se resolvía en lo siguiente: la otra curandera había manipulado fuerzas que tocaron "algún centro del cuerpo” (del interior del cuerpo

\footnotetext{
4 El encuentro entre Víctor y la curandera se sucedió como una conversación en la que el esperaba que a partir de su relato ella intentara algún diagnóstico. Los poderes de la mujer dependen de un don personal que Víctor y otros moradores del barrio le reconocen y que se confirma a través de la performance que ella realiza en la consulta sin que esto sea reconocido por los actores como un ceremonial o ritual.
} 
de Víctor). Pero este centro y las fuerzas derivadas no se comportaron de acuerdo a lo deseado por la curandera aliada a la pretendiente sino produciendo las desgracias de Víctor.

Este es un ejemplo en que puede observarse la vigencia de la concepción monista de la persona. Las notas características de esta concepción, que pueden desgajarse del trayecto que va desde la percepción de la enfermedad por parte de Víctor hasta su cura, son las siguientes:

a) La existencia de un plano de relaciones interpersonales, y una moralidad que le es propia como paño de fondo que se actualiza en la etiología del complejo de síntomas ${ }^{5}$ b) la circularidad permanente entre lo físico y lo anímico de manera que junto a, c) la fluidez que caracteriza al vaivén de causa efectos entre uno y otro medio hacen que d) lo físico y lo anímico se tornen planos derivados e irreductibles a la unidad en la que están imbricados.

En términos de este lenguaje que subyace a la práctica de cura lo corporal (la falta de fuerzas que afronto Víctor) es un momento y la actualización de una vivencia (en un sentido moral, mental y espiritual) más que una materialidad objetivada y separada sobre la que se puede operar desde fuera del conjunto de elementos valorativos y simbólicos en las que emerge.

En términos de esta comprensión de lo que es patológico y lo que es terapéutico los hechos no son connotados como milagrosos. ${ }^{6}$ Aun cuando en el momento de ejercer su terapia, la curandera haya invocado el poder de entidades sobrenaturales explicaciones apelan a fuerzas y energías que dan razón de los estado de morbidez o salud y a los poderes que las movilizan. Pero, si la cura se estructura como un saber no quiere decir que la etiología de la enfermedad y la propia cura, como lo hemos dicho, no abarquen un plano espiritual. Lo que sucede es que en estas representaciones los factores "sobrenaturales” solo tienen ese carácter entre comillas por que la división entre sobrenatural y natural es de otro sistema de clasificaciones. De la misma manera que el curanderismo es religioso sin proponerse a sí mismo como religión

\footnotetext{
5 El análisis que estamos realizando se basa en la siguiente interpretación: en el circuito etiológico es imposible separar el trabajo de brujería de la intención aviesa que, en términos de los imperativos culturales a los que Víctor adhiere, guían a la pretendiente y, también, parcialmente, conmueven al propio Víctor. Mas especificamente debe decirse que esto implica al aspecto relacional de la concepción monista.

6 Tal como se verá más adelante esta noción adquiere un contenido específico en el diferente en el marco de la concepción dualista que abordaremos en este trabajo.
} 
el plano espiritual es parte de la unidad más profunda en la que hunden sus raíces los fenómenos patológicos (así como se asocia a una concepción relacional el monismo es cosmocéntrico ${ }^{7}$ ).

La densidad propia de estas categorías y, sobretodo, su vitalidad se comprueban en su capacidad de articular el sentido de las prácticas religioso-terapéuticas en el contexto de algunas iglesias pentecostales locales. Desde sus inicios en el hemisferio norte y, mucho más, desde su implantación latinoamericana el discurso pentecostal plantea una opción terapéutica presentando la salud como una de las bendiciones que se pueden recibir de la divinidad. Los términos en que se articulan la práctica y el discurso de cura divina son una oportunidad para verificar la diversidad de expresiones que alcanza la concepción holista y, al mismo tiempo, desplegar sus matices.

En la teología tradicional pentecostal la imposición de manos es, al mismo, tiempo un gesto de identificación con el modo de obrar de Jesús y una forma de transmitir una bendición genérica y generalizada. La frente del creyente recibe el poder de Dios que se infunde a toda su vida. Entre algunos de los pastores pentecostales de la zona se desarrolla, en cambio, una concepción energética de la bendición. La invocación a Dios hace descender un poder de cura que desciende sobre el creyente. Pero además no resulta indiferente la zona del cuerpo en que esto se haga ya que la aflicción está localizada. A veces se aplica la bendición siguiendo la orientación que proporcionan las señales de dolor para situar el lugar preciso. En caso más extremos se plantea el criterio de que la aflicción se localiza, de acuerdo a una lógica moral, según tipos de pecado. Así para un pastor de la zona la falta de oración lleva al dolor de garganta y la falta de entrega a dios lleva al estreñimiento. La bendición se aplica, entonces, en el lugar del síntoma físico, pero se acompaña de la exhortación a corregir la actitud de pecado.

Esta misma caracterización de la bendición como fuerza sanadora transforma la doctrina del bautismo. En la tradición protestante esta es una práctica reservada a quien puede elegir y tiene conciencia. Para algunos pastores de la zona es, en cambio, algo que hay que hacer lo más rápido posible garantizar la

\footnotetext{
Siguiendo la expresión de Birman (1995) y la caracterización de la esfera de la religiosidad popular en términos de la vigencia más plena del creer como forma del lazo social a la que la segmentación moderna perfora y ocluye (Ver Duarte, 1982).
} 
salud de los recién nacidos. Si bien esta actitud puede ser determinada por la necesidad de consolidar la masa de creyentes es obvio que el argumento no es casual: supone, una vez más, la lógica según la cual la práctica religiosa organiza un intercambio de fuerzas entre el plano espiritual y la unidad biológica mínima. Esta asimilación del pentecostalismo a las imágenes que sostienen la unidad de las categorías físicas y morales se expresa, también, en un caso indudablemente extremo pero revelador de la lógica presente en los ejemplos antedichos: el caso de una pastora que para hacer fluir el Espíritu Santo en la iglesia daba de mamar de sus pechos a los hijos de sus hermanas de fe. Menos extremo es el caso de una mujer que pertenece a una iglesia evangélica y ha renovado sus métodos de curadora del empacho. Si bien sus procedimiento de manipulación física y espiritual de las fuerzas que alteran el proceso digestivo son los mismos que los de los curanderos su variación es la supresión de la invocación de los santos y la virgen para dirigirse exclusivamente a Cristo. En vez de acudir a las mediaciones, casi politeístas del catolicismo popular, el holismo de esta mujer se vuelve monoteísta. Pero aún aquí la concepción de una energía y una mediación entre todos los planos de relación humana se mantiene: el tratamiento, según esta mujer "mejora por que la bendición llega mejor”, por que la disminución de las mediaciones deja circular más libre y directamente la bendición. ${ }^{8}$

Dualistas y Trinitarios: Si por una convención aceptamos que la anterior es una noción tradicional las dos configuraciones que siguen puede ser llamadas modernas por que sus temas y su lenguaje remiten a la presencia de instituciones que operan en dirección a la fragmentación, la individualización, la racionalización, propias de la modernidad (aunque una serie de mediaciones propias de la subcultura de nuestros informantes hagan que sus efectos fragüen en un complejo de temas y actividades que no se iguala al que es propio de los grupos sociales más nítidamente afinado con la modernidad). La primera hace presente la influencia y procesamiento de las ideologías

\footnotetext{
8 La coincidencia entre una parte de los curanderos y algunos pastores deriva de la convergencia alrededor de las nociones que describen los procesos de cura y la noción de persona contenida en ellos. Ella se demuestra, también, en el hecho de que los usuarios no hacen distinción a no ser por cuestiones exteriores a lo esencial de la concepción monista. Así hay fieles y usuarios que diferencian esas prácticas pentecostales de las de los curanderos por el hecho de que estas últimas son “ocultistas” en un sentido que se define por contraposición al de las primeras que se hacen a cielo abierto.
}

Horizontes Antropológicos, Porto Alegre, ano 4, n. 9, p. 29-45, out. 1998 
modernamente elaboradas por el catolicismo ${ }^{9} \mathrm{y}$, en otro nivel, por los modelos científicos, especialmente médicos. La segunda registra el influjo de la expansión de las instituciones que introducen la noción de salud mental y los discursos psicologizantes.

Osvaldo es un buen ejemplo de la primera variación que, respecto de las nociones monistas, queremos poner en evidencia. Ha sido alcohólico y ha llevado adelante un proceso que incluyo su pasaje por Alcohólicos Anónimos y el Movimiento de Renovación Carismática. Al intentar un balance de experiencia terapéutica, computando tanto su cura de la adicción como las consecuencias hepáticas, dice:

... estoy bien espiritualmente hablando. Pero yo no puedo olvidarme que he llevado una vida de desarreglos que me ha destruido el cuerpo. Es que hay algo que es terrible pero es así: Dios perdona pero la naturaleza no.

La cura se ha realizado solo parcialmente. Es que en la concepción dualista existen dos órdenes de experiencia totalmente separados. De un lado el orden de los afectos, los pensamientos las emociones y, fundamentalmente, la relación con el plano trascendental en que se ubica Dios. Del otro el cuerpo que es el soporte efímero de una entidad espiritual y que respecto de la persona santa que encarna ahora Osvaldo es una entidad objetivada y casi externa.

Cierto es que esta es una presentación extrema de una forma ya clásica de construir la experiencia el occidente moderno. Pero la forma exasperada que aparece en el caso citado es el vector que debe presumirse para entender los ejemplos subsiguientes. A través de ellos podrá apreciarse que esta no es solo una declaración sino una formación simbólica que ejerce una constricción sobre las prácticas.

Es lo que se ve en el surgimiento polémico de estas categorías en este ejemplo proveniente del campo pentecostal. En una de las frecuentes discusiones sobre la necesidad y el momento de acudir a los médicos en vez de a la sanidad divina, una situación en si bastante significativa, pude observar la

9 Nos referimos las tendencias al desencantamiento que el propio catolicismo activa y no, aunque lo suponemos en este trabajo, a su presencia como vector que en la larga duración histórica es agente de la individuación. Sobre este punto ver Duarte y Giumbelli (1994). 
emergencia de la posición dualista. Surgía oponiéndose a un pastor que defendía a ultranza el recurso a la sanidad divina: ${ }^{10}$

... yo entiendo que nosotros tengamos que protegernos, que no podemos hacer cualquier cosa por que entonces nos pueden hacer un juicio por ejercicio ilegal de la medicina. Pero asi es como que no creemos en la sanidad divina (J. Pastor Iglesia Pentecostal Viña del Señor).

Un creyente que termino migrando de la iglesia de este pastor a otra del barrio afirma la posición en la que se introducen las concepciones dualistas:

Yo no estoy de acuerdo con J. Nosotros no somos solamente espirituales. Nosotros somos carnales y espirituales. Y entonces nosotros estamos en el mundo y tenemos un cuerpo y tenemos que cuidarlo lo mejor posible (Daniel, Iglesia Pentecostal Sermón del Monte)

El dualismo que establece cuestiones del cuerpo separadas de las espirituales, y privilegia al saber médico, se manifiesta en la iglesia de Daniel en los más diversos planos. Desde la colaboración financiera con la sala barrial de primeros auxilios hasta el control de las visitas médicas de las familias por parte del pastor. Para ellos el recurso médico y las acciones colectivas destinados a gerenciarlos son tan importantes como los cultos.

En el campo en que dominan estas nociones la idea de milagro adquiere una connotación especifica que se aprehende tomando en cuenta los dos planos en que el dualismo se despliega y escinde. Haciendo un esquema de esa división podremos comprender mejor el dualismo. De un lado lo espiritual en que, sin distinguirse o sin segmentarse, aparecen lo ideal, lo mental y lo divino- el alma humana y el Espíritu Santo-. Del otro el cuerpo que se vuelve explicable y objetivo a partir de divulgación de las nociones científicas.

De una parte el milagro es un tipo de hecho absolutamente inexplicable. No depende de ningún saber explicitable como el que es operado en la

${ }^{10}$ La posición del pastor, por su forma de operar la sanidad divina podría caracterizarse como monista. Dualismo-monismo es el par que se enfrenta en la situación etnográfica particular que sirve para datar la posición dualista. Pero no hay obstáculo para considerar posibles situaciones en las que la posición dualista surgir sin contraposiciones o, polémicamente, frente a una opción dualista/fatalista que, por otras razones, rehuiría del recurso médico. 
concepción holista, refiere a fuerzas y lugares del cuerpo que articulan un flujo físico-moral y tiene al curador como parte. Aquí el milagro solo precisa de dones que, coherentemente con la lógica dualista, son exclusivamente espirituales (la esperanza, la transparencia, el poder de oración, de comunicarse con dios para que el intervenga sobre el cuerpo del afligido) y sirve para desencadenar el supremo arbitrio de Dios que es quien decide curar y sabe como.

Pero esa misma definición del milagro se hace paradojalmente dependiente de la ciencia como principio de cura. El milagro trabaja en los puntos ciegos de la ciencia ("los médicos no sabían que hacer”, "los médicos ya lo habían dado por muerto" son aseveraciones comunes en los relatos) por lo que es preciso hacer jugar una suposición de hasta donde llega la ciencia. Y al mismo tiempo la ciencia valoriza el milagro ya que cuanto más difícil para la ciencia, más importante y más improbable es el milagro. Así en medio de un culto en el que un fiel solicito que se orara para que su hijo saliera de la cárcel y se curase de sida la pastora intervino y dijo:

Es muy posible que salga pero lo de la enfermedad sería un bruto milagro. No es una conjuntivitis (M. Pastora de la Iglesia Pentecostal Sermón del Monte).

Pero si los milagros tienen posibilidades que se evalúan en términos propios de la ciencia es por que, como contrapartida del espiritualismo, puede observarse una especie de materialismo popular que se prolonga en dos sentidos. Primero como explicación materialista de los milagros y, luego, como prescindencia de cualquier mediación que remita a lo tradicional y o lo espiritual.

Del primer sesgo dan testimonio las frecuentes explicaciones que intentan desmitificar milagros adjudicándoles el carácter de sugestión y haciendo intervenir un elemento mental que a veces reconduce a una concepción holista ${ }^{11} \mathrm{y}$, otras, se abre a la psicologización de la que hablaremos en el próximo apartado. Del segundo dan cuenta casos como el de Cesar en el que el privilegio otorgado a lo que proviene de la ciencia es tan grande que determina una posibilidad de rechazo de los recursos tradicionales de cura y sus categorizaciones de la persona. Cesar se quejó durante varios meses que coincidieron

${ }^{11}$ Es importante acotar esto por que no se nos escapa que el mero uso de un lenguaje "científico" puede estar dejando hablar a un código similar al nervioso. Este o podría ser el caso de ciertas apelaciones que he registrado y que están referidas al stress y, aun, a las enfermedades psicosomáticas.

Horizontes Antropológicos, Porto Alegre, ano 4, n. 9, p. 29-45, out. 1998 
con mi estancia en campo de una molestia que le nublaba el ojo derecho. Se sentía abrumado por que no tenía dinero para ir a un buen oculista que le solucionara lo que suponía que era un problema de presión ocular y, al mismo tiempo, decía no ser "tan gil”" ${ }^{12}$ como para confiarse a pastores o curanderos. ${ }^{13}$

Pero si la combinación del prestigio de la ciencia con la actitud racionalizante de algunas iglesias transforma la idea de milagro y deja un cuerpo maquinizado y objeto de la ciencia, la diseminación de las prácticas y discursos vinculados a la psicología induce una recomposición del campo de las prácticas de cura que acrecentando las alternativas tanto (como redefiniendo las existentes) conlleva la formulación de nuevas categorías.

Una parte importante de los informantes ha desarrollado prácticas de cura en el campo de las psicoterapias. ${ }^{14} \mathrm{Y}$ a mayor parte de ellos (y que por la orientación de mi investigación pertenecen a algún agrupamiento religioso) explicitaban una nueva división de los planos de la experiencia. Para ellos es como si hubiera habido un plano que se oponía o se diferenciaba de lo material y lo corporal que ahora resulta subdividido. Así aparece de un lado un lado lo que es psicológico y:

son cosas mías (Nora), tienen que ver con los conflictos, con las trabas que uno tiene para hacer cosas (Malvina). ${ }^{15}$

De otro se encuentra el espiritual que tienen que ver con:

las fuerzas que vos necesitas de Dios para poder seguir adelante (Malvina), con la protección que cada uno pide para su familia y su casa (Nora).

${ }^{12}$ En portugués este término puede traducirse como "otário".

${ }^{13}$ Esta cuestión entra, parcialmente, en la lógica de la pérdida de valor social de los recursos tradicionales. En este sentido una reciente etnografía muestra como una familia de desempleados del Gran Buenos Aires prefiere, contra toda racionalidad económica, los fármacos sómniferos a los tés calmantes considerados sustitutos para pobres (Prelorán, 1995).

${ }^{14}$ Entre cuarenta informantes que se localizan en los segmentos de edad de entre 20 y 50 años siete encararon tratamientos psicoterapeuticos de los que tuve noticia por la importancia que le adjudicaban en su historia personal. Además es preciso consignar el alto grado de difusión que tienen materiales audiovisuales y literarios que refieren a las practicas psi y que, teniendo en cuenta la expansión de las experiencias psicoterapéutica, hallan condiciones de interpretación que divergen de la ideología holista.

${ }^{15}$ Nora y Malvina son, respectivamente, una fiel católica en transición al pentecostalismo y Malvina es católica. 
Para entender el contenido del plano psicológico debe entenderse que en esta división tripartita su desarrollo es paralelo al de una orientación centrada en la actividad del sujeto y, por lo tanto, en un giro individualizante. Este se percibe claramente en la reflexión de una informante que reelabora en una clave subjetivista uno de los temas centrales de la consulta al curandero como el del "cierre de caminos" (que remite a los "trabajos” y trabas que le son efectuados a un sujeto para perjudicarlo ${ }^{16}$ ):

... uno siempre está pensando en los caminos que se le cierran pero no se pone a pensar en lo que uno hace para que esos camino se le cierren. Por que uno tiene que darse cuenta que tiene responsabilidades, no todo es lo que te hacen los otros, que vos mismo con tu actitud te cerrás los caminos (Pequi, Vidente de Villa Libertad).

La orientación ego-referencial también se sustancia en un discurso que, además, ilustra otro ítem del plano psicológico. El autoconocimiento, la postulación de una interioridad portadora de la verdad subjetiva: ${ }^{17}$

La terapia me ayuda a crecer como persona. Me sentia mal y quería comprender por que me pasan estas cosa (Sandra, miembro de la Iglesia Católica de Villa Independencia).

El plano psicológico también está asociado a una específica valoración de las potencialidades terapéuticas de la palabra, la conversación la posibilidad de decir y escuchar. En la palabra se deposita la posibilidad de una nueva conciencia (él "darse cuenta de cosas que uno no conoce de uno mismo” con el cual una de mis informantes definía la especificidad de su terapia) mediada por una relación que no implica un orden sobrenatural con el que es preciso reconstruir puentes o dar testimonio de permanente inclusión.

${ }^{16}$ Obsérvese que este es el mismo tópico que estaba en juego en la dolencia de Víctor. En la diferencia entre un discurso y otro puede percibirse claramente la diferencias entre un centramiento en el plano de las relaciones y uno que se realiza sobre el ego.

17 En este sentido Blanco (1996) hace observaciones semejantes sobre la las características actuales de la cultura terapéutica de los sectores populares. En ese plano detecta una torsión a favor de alternativas terapéuticas y esotéricas destinadas mas a la autocomprensión que al conocimiento del destino.

Horizontes Antropológicos, Porto Alegre, ano 4, n. 9, p. 29-45, out. 1998 
Pero la fe y la terapia, lo psicológico y lo espiritual no se contradicen. Tienen canales diferenciados:

La terapia no cree en nada. La fe me da fuerza espiritual y la terapia me da fuerza personal, me da valor como persona. La fe me da fuerza al corazón (Nora).

Pero tampoco lo espiritual y lo psicológico están escindidos o son equivalentes. En este sentido los contenidos del plano espiritual están separados pero suplementan y engloban el plano psicológico. El plano espiritual es presentado como el reino de la inmediatez, el contacto directo con Dios, con el principio vital que este encarna. Por que, como citamos, a través de ese principio se asegura la casa, la familia, el orden del mundo social que circunda al yo (ya efecto de la psicología), el plano espiritual se presenta como un manto protector que se ha alejado merced a la germinación de un yo. Este lo pone a distancia al emerger como un principio inasimilable y, aún, le impone una transformación individualizante a través de una definición romántica en la que las relaciones con la divinidad, son de corazón a corazon, de transparencia del creyente frente a Dios en una relación íntima.

La diferencia que esto presenta con las ideologías que auto-presentadas como holistas, y que caracterizan a las experiencias "new age” de las clases medias, pone de manifiesto la especificidad de la estructura tripartita expuesta aquí. Mientras en la new age se trata de la recuperación de un énfasis místico, y de la tentativa de reconciliarlo con el patrimonio psicologista heredado y reproducido por décadas, aquí se trata de dar cuenta de la activación de un núcleo psicologista de reciente data. Activación que resulta conflictiva ya que debe lidiar con los estigmas de la locura y con el cuestionamiento de líderes religiosos que muchas veces sienten afectada su jurisdicción. La psicologización y la tripartición entre los informantes que cito esta más acá de la ruptura con el holismo mientras que las concepciones new age están más allá aun cuando su horizonte (imposible) sea el retorno.

Este es el contexto en que la psicologizaciónse tiende puentes con el monismo popular y la mediación entre lo físico y lo moral se reactiva. La combinación entre las referencias intimistas, psicologizadas y subjetivadas y categorías relativas a un sufrimiento que se irradia a todo el ser son la base que hace posible esa conexión. Por otra parte no debe pensarse que el giro 
individualizante se trasunta en el abandono de los imperativos culturales que en plano relacional definen el valor supremo del proyecto familiar y una definición complementaria y jerárquica de los papeles familiares. Así una de las mujeres pentecostales que entendía que su conversión al pentecostalismo se había dado por que según ella le había ayudado a mejorar su autoestima ${ }^{18} \mathrm{ca}-$ nalizaba el bienestar recuperado hacía la recomposición de su propia familia y a la compensación de las faltas que había cometido a su papel de mujer-esposa (que habían sido realizadas con su depresión).

Hay otro caso emblemático de las relativizaciones monistas de la concepción trinitaria que se inscribe en las consideraciones anteriores. La pastora de una iglesia pentecostal recibe a una mujer que llega desesperada por la fiebre de su hijo. La pastora que conoce algo de la situación familiar de la recién llegada realiza un breve interrogatorio en el que consigue ampliar su información (confirmo que el marido de la mujer había vuelto a tomar y a pegarle). Luego emite un consejo basado en lo que ella considera el principal problema:

el chico no está enfermo, la que está enferma sos vos. Tenes que plantarte con tu marido. No agaches la cabeza. Cuando lo hagas el chico se va a mejorar.

La madre del niño enfermo esperaba otra cosa: una oración, un masaje para el empacho, la imposición de manos en el estómago. La pastora sorprendió a la mujer. Y también a mí con el relato y los fundamentos de su intervención. ${ }^{19}$ Según ella esto era algo que había aprendido con "las cosas que le habían pasado en la vida”. Su relato se remontó entonces a su propia experiencia como madre que participaba del grupo de apoyo psicoterapéutico para familiares de menores internados en el hospital público de la zona. Allí había aprendido lo importante que era darse cuenta de cómo “el manejo de la familia es importante para las enfermedades”, de que "uno puede estar haciendo cosas que no sabe lo que significan” y de lo importante que resulta que

\footnotetext{
18 Esta mujer explicitaba una conexión y una complementariedad entre su tratamiento y su conversión. En su elaboración las cosas eran así. El tratamiento le había hecho ver que "no estaba cuidando de si y si no se quería no la iban a querer”. La iglesia le había dado el afecto que ella misma no se daba. En base a la descripción de un individualismo diferente del aquí propuesto Mariz y Machado (1996) también refieren a la articulación entre un plano intrapersonal definido en forma individualista y un plano interpersonal definido de acuerdo a reglas holistas.

19 La exposición de este caso se hace sobre la base de dos relatos. El de la madre del niño y el de la pastora.
} 
“uno lo pueda ver”. Quería movilizar a la mujer para resistir los golpes de su marido sin quebrar el matrimonio y distender el hogar que enfermaba al niño.

Para la pastora el tratamiento aplicado era parecido a lo que hacía el psicólogo del hospital donde había estado con su hijo. Nosotros podríamos ver como a partir de una técnica que intenta imitar el consultorio o el grupo de terapia la pastora recicla las mediaciones físico morales en el plano relaciona atendiendo a la específica configuración familiar holista.

\section{III - Conclusión}

Victor agrupó, naturalmente, sin contradicción, los trazos de su aflicción en un mal único y, naturalmente, acudió una curandera que, con igual naturalidad acompaño su presuposición y le ayudó a tener una definición más clara de ese mal y a superarlo. El psicólogo, el médico forman parte de sus posibilidades de contacto cotidiano pero no de su stock real de recursos terapéuticos (para ese mal al menos). Osvaldo, Margarita Nora, Pequi, nacieron y vivieron en el mismo barrio, (algunos de ellos en la misma manzana que Víctor). Pero sufren diferentemente y se curan sobre la base de suposiciones diferentes que remiten a construcciones diferentes de la persona, de las divisiones que la constituyen, de las relaciones entre esos compartimientos. Las vertientes presentadas exigen, sin duda, una investigación destinada al conocimiento más profundo de su articulación y funcionamiento pero son innegables bases de la diferenciación de las formas de sufrir y recibir alivio.

A esa conclusión que recapitula lo expuesto pretendemos agregar otra que esquematiza las relaciones entre las diferentes concepciones dado que coexisten en el tiempo y, a veces, en un mismo sujeto o en una misma trayectoria.

La concepción que hemos llamado monista es la más extendida y al mismo la más difusa. ${ }^{20}$ En ese carácter guarda una relación de continente a contenido con el dualismo y la psicologización de la concepción trinitaria. Esto puede deducirse no solo del predominio numérico que tiene la primera en el conjunto de los informantes que conforman el universo de la investigación. También del hecho de que las referencias dualistas y psicologizadas tienden

${ }^{20}$ Utilizamos este término en el sentido de que encuentra poco articulada institucionalmente pero constituye un conjunto de categorías ampliamente difundidas y utilizadas. 
a funcionar en un espacio circunscripto por la presencia de un dispositivo que favorece su surgimiento. La psicologización depende de psicólogos, de lecturas. No se trata de que se enseñe y se imponga en los términos de los psicólogos, pero sí de la posibilidad de que su estimulo se ofrezca a la combinación en que la cultura de los habitantes de Villa Libertad les otorga un sentido específico. De esta forma el dualismo y el trinitarismo pueden dar paso a prácticas monistas que los reinterpretan o los disuelven en su propia matriz. En la relación de inclusión que planteamos el dualismo y el trinitarismo parecen incrustaciones en un medio monista que al mismo tiempo que los en vuele se ve transformado. Las nuevas representaciones no conforman, además, un sistema tan extenso que o pueda, punto por punto, paralelizar a la concepción monista. Esto explica los casos en que en la sincronía de un sujeto se acumula o alterna el uso de las diversas matrices (aunque es una cuestión a resolver en cada caso cual de ellas es predominante).

\section{Referencias}

BLANCO, A. La cultura terapéutica de los sectores populares. Notas para un debate. Ciencias Sociales: Boletín de la Facultad de Ciencias Sociales. Buenos Aires: Universidad de Buenos Aires, 1996.

BIRMAN, P. Fazer estilo criando gêneros: estudo sobre a construção religiosa da possessão e da diferença de gêneros em terreiros de umbanda e candomblé do Rio de Janeiro. Rio de Janeiro: Ed. UERJ, 1995.

DUARTE, L. F. D. Pluralidade religiosa nas sociedades complexas e "religiosidade" das classes trabalhadoras urbanas. 1982. Encontro Anual Anpocs, 1982.

DUARTE, L. F. D. Da vida nervosa nas classes trabalhadoras urbanas. Rio de Janeiro: Jorge Zahar Editor, 1986.

DUARTE, L. F. D. A outra saúde: mental, psicossocial, físico-moral? In: ALVES, P. C.; MINAYO, M. C. (Ed.). Saúde e doença: um olhar antropológico. Rio de Janeiro: Editora Fiocruz, 1994. 
DUARTE, L. F. D.; GIUMBELLI, E. A. As concepções de pessoa cristã e moderna: paradoxos de uma continuidade. In:ANUÁRIOANTROPOLÓGICO 93. Brasília: UnB, 1994.

DUMONT, L. Homo hierarquicus. São Paulo: Editora da Universidade de São Paulo, 1992.

MARIZ, C.; MACHADO, M. das D. C. Pentecostalismo e a redefinição do feminino. Religião \& Sociedade, Rio de Janeiro, v. 17, n. 1/2, p. 140-159, 1996.

PRELORÁN, M. Aguantando la caída. Buenos Aires: Mutantia, 1995. (Colección Testimonios).

SANCHIS, P. "O campo religioso contemporâneo no Brasil. In: ORO, A. P.; STEIL, C. A. (Org.). Globalização e religião. Petrópolis: Vozes, 1997. 